\title{
Application of Swarm Intelligent Algorithm Optimization Neural Network in Network Security
}

\author{
Hui Xia ${ }^{1}$ \\ Software College, Shenyang Normal University, Shenyang, 110034, China \\ freund_xia@126.com
}

Keywords: Traffic Detection; Swarm Intelligence Algorithm; Neural Network; Network Security

\begin{abstract}
A network traffic detection model based on swarm intelligent optimization neural network algorithm is proposed in this paper. QAPSO algorithm is used to optimize the basis function center and base function width of RBF neural network, and the connection weights of the output layer and the hidden layer as well. This paper analyzes the detection model studied in this paper by an example, and use the collected data to train the network traffic identification system and test its performance. The comparison between the proposed method and the conventional PSO algorithm based on the HPSO algorithm shows that the proposed method has faster recognition speed and better recognition accuracy, and avoids the problem of falling into the local optimal solution. Situation.
\end{abstract}

\section{Introduction}

With the continuous development and popularization of Internet technology, Internet applications and services in increasing types, in order to improve network security, protection of Internet users, companies and government departments, such as property and interests of the need for efficient network traffic monitoring $[1,2]$.

RBF neural network has a strong nonlinear fitting ability, that is, non-linear mapping ability, as well as self-learning ability, while easy to computer implementation, and thus in the network traffic detection network security has been widely used. However, the performance of RBF neural network depends on the selection of network parameters. The traditional RBF neural network parameters are usually selected by experience or random, so the performance of RBF neural network has strong randomicity [3, 4].

In recent years, the swarm intelligence optimization algorithm has been developed and applied widely. Particle Swarm Optimization (PSO) is a group swarm optimization algorithm with global optimization, fast modeling speed and high convergence efficiency. However, using PSO algorithm, The convergence rate and global optimization ability of the network still can not reach equilibrium and other issues [5-7]. Therefore, this paper investigates a quantum adaptive particle swarm optimization algorithm (QAPSO), which optimizes the base function center $C_{i}$, the width $\sigma_{i}$ of the basis function and the connection weight $w_{i}$ between the output layer and the hidden layer of the RBF neural network.

\section{A Neural Network Algorithm Based on Swarm Intelligence Optimization}

The QAPSO algorithm is divided into four parts, which are initial population, estimating evolution state, adaptive control parameter and dealing with variation [8].

1) Initialize the population

Quantum bits are two-state quantum systems that are different from traditional bits, because they can fall in a linear combination of $|0\rangle$ and $|1\rangle$, called the superposition state, as follows:

$$
|\psi\rangle=\alpha|0\rangle+\beta|1\rangle
$$

In the formula, $\alpha$ and $\beta$ are as follows:

$$
|\alpha|^{2}+|\beta|^{2}=1
$$


The encoding of the current position is represented as follows:

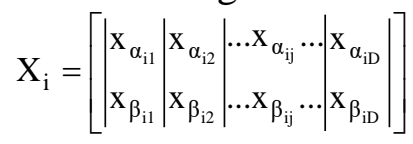

Where $x_{\alpha_{i j}}$ is the probability amplitude of $x_{i j}$ for quantum state $|0\rangle$, the initial value is $\sqrt{1 / 2}$; $x_{\beta_{i j}}$ is the probability amplitude of $x_{i j}$ for quantum state $|1\rangle$, and the initial value is $\sqrt{1 / 2}$. It can be shown that the probability of each particle for the states $|0\rangle$ and $|1\rangle$ is expressed as:

$$
\begin{aligned}
& X_{i \alpha}=\left[x_{\alpha_{i 1}}, x_{\alpha_{i 2}}, \ldots, x_{\alpha_{i D}}\right] \\
& X_{i \beta}=\left[x_{\beta_{i 1}}, x_{\beta_{i 2}}, \ldots, x_{\beta_{i D}}\right] \\
& X_{\alpha_{i j}} \text { and } X_{\beta_{i j}} \text { of each particle are the solutions of the optimization problem, and thus the } \\
& \text { 2) Estimate the state of evolution } \\
& \text { The average distance of each particle relative to other particles is estimated to be: } \\
& d_{i x}=\frac{1}{N-1} \sum_{j=1, j \neq i}^{N} \sqrt{\sum_{k=1}^{D}\left(x_{i k}-x_{j k}\right)^{2}}
\end{aligned}
$$
ergodicity of the optimization algorithm search is expanded.

The average distance of each particle relative to other particles is estimated to be:

The average velocity difference for each particle relative to other particles is estimated to be:

$$
d_{i v}=\frac{1}{N-1} \sum_{j=1, j \neq i}^{N} \sqrt{\sum_{k=1}^{D}\left(v_{i k}-v_{j k}\right)^{2}}
$$

Particle position and velocity together determine the particle trajectory. The average trajectory difference $d_{i c}$ of the particles is determined by the mean distance between the particles and the average velocity difference, and the average trajectory difference $d_{i c}$ is a mixture of the mean distance between the particles and the average velocity difference,the representation is:

$$
\begin{aligned}
d_{i c} & =d_{i x}+\rho_{X_{i} Y_{i}} d_{i v} \\
\rho_{X_{i} Y_{i}} & =\frac{E\left(X_{i} V_{i}\right)-E\left(X_{i}\right) E\left(V_{i}\right)}{\sqrt{E\left(X_{i}^{2}-E_{2}\left(X_{i}\right)\right)} \sqrt{\left.E\left(V_{i}^{2}\right)-E_{2}\left(X_{i}\right)\right)}} \\
& =\frac{\sum_{j=1}^{D} x_{i j} v_{i j}-\frac{1}{D} \sum_{j=1}^{D} x_{i j} \sum_{j=1}^{D} v_{i j}}{\sum_{j=1}^{D} x_{i j}-\frac{1}{D}\left(\sum_{j=1}^{D} x_{i j}\right) \sqrt{\sum_{j=1}^{D} v_{i j}{ }^{2}-\frac{1}{D}\left(\sum_{j=1}^{D} v_{i j}\right)^{2}}}
\end{aligned}
$$

The average trajectory difference of the optimal particle and other particles is $d_{c g}$, and the evolution factor $f_{c}$ of the average locus difference $d_{c g}$ is expressed as:

$$
f_{c}=\frac{d_{g}-d_{\text {min }}}{d_{\text {max }}-d_{\text {min }}} \in[0,1]
$$

Where $d_{\max }$ is the maximum value of the track difference and $d_{\min }$ is the minimum value of the track difference.

3) Control parameter adaptation

The global and local search ability of the particle swarm are balanced by the inertia weight. Therefore, according to the acceleration coefficient $c_{1}$ and $c_{2}$ adjustment strategies shown in Eq. (11) and Table 1, adjust the acceleration coefficient in each state.

$$
w_{c}\left(f_{c}\right)=\frac{1}{1+1.5 e^{-2.6 f_{c}}} \in[0.4,0.9]
$$

Table 1 slightly increased, slightly reduced by the formula (12) to determine: 


$$
c_{i}(g+1)=c_{i}(g) \pm \delta, \quad i=1,2
$$

In the formula, $\delta$ is a random number of 0.05 to 0.1 .

4) Handling Variations

In order to converge the probability of the global best particle in convergence, the quantum bit of the global best particle will be changed according to the mutation probability. Quantum bits are subjected to mutation processing using quantum non-gates, denoted as:

$$
\left[\begin{array}{ll}
0 & 1 \\
1 & 0
\end{array}\right]\left[\begin{array}{c}
p_{\alpha_{i}} \\
p_{\beta_{i}}
\end{array}\right]=\left[\begin{array}{c}
p_{\beta_{i}} \\
p_{\alpha_{i}}
\end{array}\right], 1 \leq i \leq D
$$

\section{Test results}

In order to verify the performance of the network traffic detection model based on QAPSO optimized RBF neural network, C \# program based on Libsvm software package was used to collect, calculate and classify the network traffic with Matlab R2014. The network traffic detection types are shown in Table 2.

Table 1.The acceleration coefficients $c_{1}$ and $c_{2}$ adjust the strategy

\begin{tabular}{l|l|l}
\hline$f_{c}$ Value state & $c_{1}$ adjust strategy & $c_{2}$ adjust strateg \\
Detection & increase & decrease \\
open up & Slightly increased & Slightly decrease \\
convergence & Slightly increasedsharing & Slightly decreas \\
Leap out & decrease & increase \\
\hline
\end{tabular}

Table 2.Network traffic detection type

\begin{tabular}{l|l|l|l}
\hline Numbering & category & Numbering & category \\
A & WWW & G & SERVICES \\
B & P2PAudio and video & H & INTERACTIVE \\
C & P2P file sharing & I & DATABASE \\
D & P2P instant messaging & G & ATTACK \\
E & BULK & K & MULTIMEDIA \\
F & MAIL & L & GAMES \\
\hline
\end{tabular}

Using the conventional PSO optimization algorithm and HPSO optimization algorithm to optimize the RBF neural network and establish the same network traffic detection model, using the same training data samples for training, using the same test data samples for performance testing. The parameters of the conventional PSO optimization algorithm are space dimension D $=24$, particle number $\mathrm{N}=30$, maximum iteration number $t_{\max }=200$, connection weight $\mathrm{w}=0.9 \sim 0.4$, acceleration coefficients $c_{1}$ and $c_{2}$ are all 2. The parameters of the HPSO optimization algorithm are: space dimension $\mathrm{D}=24$, particle number $\mathrm{N}=30$, maximum iteration times $t_{\max }=200$, connection weights $w=0.8 \sim 0.2$, acceleration coefficients $c_{1}$ and $c_{2}$ are $2.5, V_{\max d}=0.5 \times$ Range.The parameters of the QAPSO algorithm are the dimension D $=24$, the number of particles $\mathrm{N}=30$, the maximum number of iterations $t_{\max }=200$, the connecting weights $w=0.8 \sim 0.2$, the acceleration coefficients $c_{1}$ and $c_{2}$ are 1.5-2.5, $V_{\max d}=$ Range, $r_{1 d}$ and $r_{2 d}$ are random numbers between 0 and 1 .

The fitness function of the detection model is the mean square error of the RBF neural network.

$$
F=\frac{1}{n} \sum_{i=1}^{n}[\hat{x}(i)-x(i)]^{2}
$$


Table 3.The detection accuracy and the feedback rate of the three detection models(\%)

\begin{tabular}{l|l|l|l|l|l|l}
\hline \multirow{2}{*}{ category } & \multicolumn{2}{l|}{ QAPSO Algorithm } & \multicolumn{2}{l|l}{$\begin{array}{l}\text { HPSO algorithm } \\
\text { Feedback }\end{array}$} & $\begin{array}{l}\text { Accuracy } \\
\text { Feedback }\end{array}$ & \multicolumn{2}{l}{ Accuracy } & \multicolumn{2}{l}{ Foedback } & Accuracy \\
A & 95.88 & 97.31 & 91.86 & 93.60 & 87.00 & 88.45 \\
B & 94.22 & 95.96 & 91.74 & 92.77 & 88.93 & 91.09 \\
C & 89.00 & 91.00 & 85.65 & 88.58 & 82.85 & 84.31 \\
D & 94.50 & 95.71 & 90.79 & 93.31 & 87.72 & 89.65 \\
E & 95.59 & 97.98 & 93.13 & 95.57 & 90.88 & 93.27 \\
F & 93.52 & 95.50 & 88.61 & 89.77 & 84.09 & 86.98 \\
G & 91.94 & 93.10 & 87.93 & 88.97 & 84.40 & 86.44 \\
H & 93.16 & 94.33 & 89.86 & 91.77 & 85.60 & 88.47 \\
I & 91.89 & 93.30 & 88.69 & 90.25 & 84.98 & 86.40 \\
J & 92.01 & 93.13 & 88.55 & 90.67 & 85.28 & 88.12 \\
K & 95.78 & 98.20 & 93.26 & 94.82 & 89.68 & 90.78 \\
L & 90.39 & 93.15 & 85.40 & 87.39 & 81.10 & 84.07 \\
\hline
\end{tabular}

Where $n$ is the number of network traffic data; $x(i)$ is the detection result of the i-th network traffic detection data; $x(i)$ is the actual result of the ith network traffic detection data.

Figure 1 shows the fitness convergence curve of the network traffic detection model using the conventional PSO optimization algorithm, the HPSO optimization algorithm and the RBF neural network with the QAPSO optimization algorithm.

It can be seen from Fig. 1 that the number of iterations of the fitness function converges to the stable value is 171 times and the HPSO optimization algorithm uses 112 times. The QAPSO optimization algorithm used in this paper only uses 7 times. At the same time, the QAPSO optimization algorithm studied in this paper has lower convergence value and fitness value is the training error of RBF neural network. So the smaller the fitness function is, the smaller the training error of RBF neural network is, the better the performance is. Therefore, the QAPSO optimization algorithm proposed in this paper has faster convergence speed and higher convergence precision than the other two PSO optimization algorithms, which greatly improves the generalization ability of RBF neural network. The experimental data were identified using the QAPSORBF detection model and the detection model of the conventional PSO and HPSO optimized RBF algorithm. Table 3 for the three detection models of detection accuracy and feedback rate comparison. Figure 2 shows the average detection rate and feedback rate of the three models.

According to the data in Table 3, we can see that the QAPSORBF detection model studied in this paper has good recognition accuracy and feedback rate for 12 types of network services and applications. The average recognition accuracy is $92.81 \%$, which is higher than HPSO-RBF The average recognition accuracy of the algorithm is 3.49\% higher than the average recognition accuracy of the PSO-RBF algorithm. The average recognition rate of the QAPSORBF algorithm is $94.81 \%$, and that of the HPSO RBF algorithm is $3.51 \%, 7.28 \%$ higher than the average recognition rate of the PSORBF algorithm. It can be shown that compared with other particle swarm optimization algorithms, the QAPSO optimization algorithm studied in this paper. 


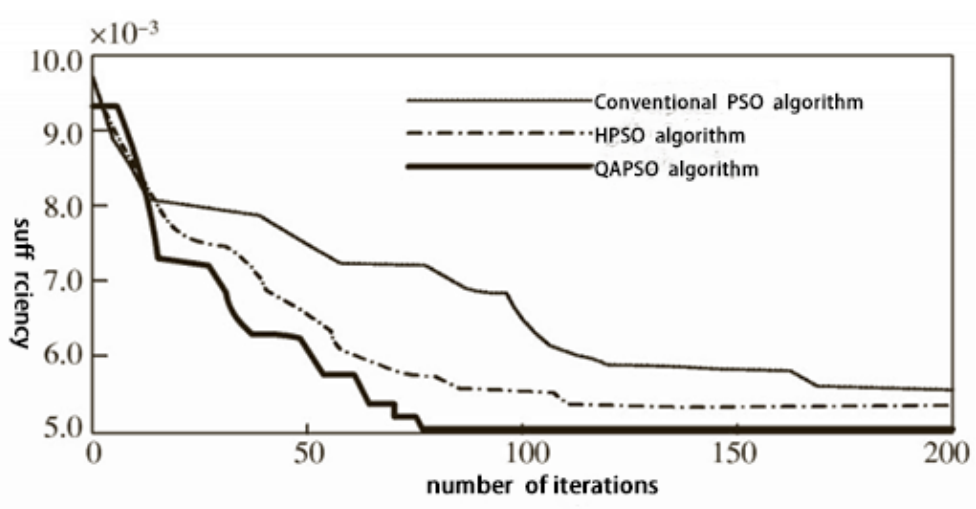

Fig.1. Frequency with the SNR curve

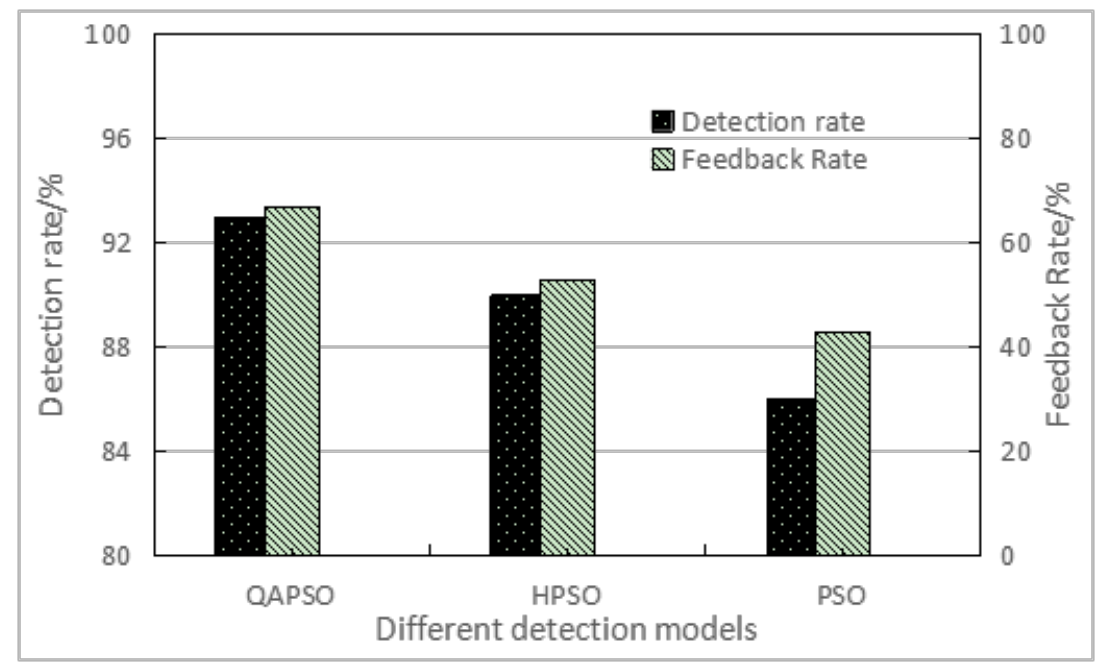

Fig.2. Comparison of average detection rate and feedback rate of three models

\section{Conclusion}

This paper studies a network traffic detection model based on swarm intelligence optimization neural network algorithm. Experimental results show that, compared with other PSO algorithms, the QAPSO optimization algorithm is still active after several iterations, jumping out of the local optimal solution and having a very significant effect on the global search ability of the optimal value the convergence speed of the algorithm is improved and the recognition accuracy is improved.

\section{References}

[1] Shi B, Li Y, Yu X, et al. A Modified Particle Swarm Optimization and Radial Basis Function Neural Network Hybrid Algorithm Model and Its Application[C]// Wri Global Congress on Intelligent Systems. IEEE Computer Society, 2009:134-138.

[2] Xu F, Fang J C. Multiobjective robust filtering algorithm for integrated navigation system based on convex optimization [J]. Yuhang Xuebao/journal of Astronautics, 2009, 30(3):937-941.

[3] Zeng X Q, Liu W. Particle swarm optimization algorithm and its application in neural networks[J]. Electric Drive Automation, 2009.

[4] Li C, Liu X. An improved PSO-BP neural network and its application to earthquake prediction[C]// Chinese Control and Decision Conference. IEEE, 2016.

[5] Zhao Z, Xu Q, Jia M. Improved shuffled frog leaping algorithm-based BP neural network and 
its application in bearing early fault diagnosis[J]. Neural Computing and Applications, 2016, 27(2):1-11.

[6] FENG Zhi-min, TIAN Li. SOC Prediction for Electric Vehicle Battery Based on AFSA-RBF Neural Network[J]. Journal of Chongqing Technology and Business University(Natural Science Edition), 2016.

[7] Kumari R, Sheetanshu, Singh M K, et al. Anomaly detection in network traffic using K-mean clustering[C]// International Conference on Recent Advances in Information Technology. 2016.

[8] Alexandridis A, Chondrodima E, Sarimveis H. Radial basis function network training using a nonsymmetric partition of the input space and particle swarm optimization.[J]. IEEE Transactions on Neural Networks \& Learning Systems, 2013, 24(2):219-230. 